\title{
SOME INEQUALITIES INVOLVING GENERALIZED BESSEL FUNCTIONS
}

\author{
ÁRPÁD BARICZ
}

Abstract. Let $u_{p}$ be the generalized and normalized Bessel function depending on parameters $b, c, p$ and let $\lambda_{p}(x)=u_{p}\left(x^{2}\right), x \in \mathbb{R}$. In this paper we extend to the function $\lambda_{p}$ some wellknown classical inequalities like Mahajan's inequality, Mitrinović's inequality, improvements of Jordan's inequality, Redheffer's inequality, using an adequate integral representation of the function $\lambda_{p}$ and the monotone form of l'Hospital's rule. Moreover we prove that the integral

$$
\varsigma_{p}(x)=\int_{0}^{x} \lambda_{p}(t) \mathrm{dt}
$$

is sub-additive (super-additive) under certain conditions on parameters $b, c, p$.

Mathematics subject classification (2000): 33C10, 26D05.

Key words and phrases: Bessel functions, modified Bessel functions, Mahajan's inequality, Jordan's inequality, Redheffer's inequality, Cusa's inequality, sine and hyperbolic sine integral.

\section{REFERENCES}

[1] H. AlzER, S. Koumandos, Sub- and superadditive properties of Fejér's sine polynomial, Bull. London Math. Soc., 38, (2) (2006), 261-268.

[2] G. D. Anderson, M. K. Vamanamurthy AND M. Vuorinen, Conformal Invariants, Inequalities, and Quasiconformal Maps, John Wiley \& Sons, New York, 1997.

[3] G. D. ANDERSON, M. K. VAMANAMURTHY AND M. VUORINEN, Inequalities for quasiconformal mappings in space, Pacific J. Math. 160, (1) (1993), 1-18.

[4] SZ. ANDRÁs, Á. BARICZ, Monotonicity property of generalized and normalized Bessel functions of complex order, Rocky Mountain J. Math., submitted.

[5] Á. BARICZ, Functional inequalities involving special functions, J. Math. Anal. Appl., 319, (2) (2006), 450-459.

[6] Á. BARICZ, Functional inequalities involving special functions II, J. Math. Anal. Appl., 327, (2) (2007), $1202-1213$.

[7] Á. BARICZ, Geometric properties of generalized Bessel functions, J. Math. Anal. Appl., submitted.

[8] Á. BARICZ, Functional inequalities involving Bessel and modified Bessel functions of the first kind, J. Math. Anal. Appl., in press.

[9] Á. BARICZ, E. Neuman, Inequalities involving generalized Bessel functions, J. Ineq. Pure and Appl. Math. 6, (4) (2005), Art. 126. Online: URL: http://jipam.vu.edu.au/article.php? sid=600.

[10] M. BENCZE, J. SÁNDOR, On Huygens's trigonometric inequality, RGMIA Res. Rep. Coll., 8, (3) (2005), Art. 14. Online: URL: http://rgmia.vu.edu.au/v8n3.html.

[11] CH.-P. CHEN, J.-W. ZHAO AND F. QI, Three inequalities involving hyperbolically trigonometric functions, Octogon Math. Mag., 12, (2) (2004), 592-596. RGMIA Res. Rep. Coll., 6, (3) (2003), Art. 4. Online: URL: http://rgmia.vu.edu.au/v6n3.html.

[12] L. DeBnath, C. J. ZHAO, New strengthened Jordan's inequality and its applications, Appl. Math. Lett., 16 (2003), 557-560.

[13] A. P. IUSKEVICI, History of Mathematics in 16th and 17th centuries, Moskva, 1961. 
[14] H. Kober, Approximation by integral functions in the complex domain, Trans. Amer. Math. Soc., 56, (22) (1944), 7-31.

[15] S. Koumandos, Some inequalities for the sine integral, J. Ineq. Pure and Appl. Math., 6, (1) (2005), Art. 25. Online: URL: http: //jipam.vu.edu.au/article.php? sid=494.

[16] L. LORCH, M. E. MuldoON, An inequality for concave functions with applications to Bessel and trigonometric functions, Facta. Univ. Ser. Math. Inform., 2 (1987), 29-34.

[17] A. Mahajan, A Bessel function inequality, Univ. Beograd Publ. Elektrotehn. Fak. Ser. Mat. Fiz., No. 634 - No. 677 (1979), 70-71.

[18] D. S. Mitrinović, Analytic Inequalities, Springer-Verlag, Berlin, 1970.

[19] E. NEUMAN, Inequalities involving Bessel functions of the first kind, J. Ineq. Pure and Appl. Math. , 5, (4) (2004), Art. 94. Online: URL: http: // jipam.vu.edu.au/article.php? sid=449.

[20] F. QI, Jordan's inequality: Refinements, generalizations, applications and related problems, , RGMIA Res. Rep. Coll. 9, (3) (2006), Art. 12. Online: URL: http://rgmia.vu.edu.au/v9n3.html. Bùděngshì Yājiuu Tongxùn (Communications in Studies on Inequalities), 13, (3) (2006), 243-259.

[21] F. QI, L. H. CUI, S. L. XU, Some inequalities constructed by Tchebysheff's integral inequality, Math. Inequal. Appl., 2, (4) (1999), 517-528.

[22] F. QI, Q. D. HAO, Refinements and sharpenings of Jordan's and Kober's inequality, Mathematics and Informatics Quarterly, 8, (3) (1998), 116-120.

[23] R. REDHEFFER, Problem 5642, Amer. Math. Monthly, 76,(1969), 422.

[24] J. SÁNDOR, A note on certain Jordan type inequalities, RGMIA Res. Rep. Coll., 10, (1) (2007), Art. 1. Online: URL: http: //rgmia.vu.edu.au/v10n1.html.

[25] J. SÁNDOR, Selected chapters of Geometry, Analysis and Number Theory, RGMIA Monographs, Victoria University, 2005. Online: URL: http: //rgmia.vu .edu.au/monographs.

[26] J. SÁNDOR, On the concavity of $\sin x / x$, Octogon Math. Mag., 13, (1) (2005), 404.

[27] G. N. Watson, A Treatise on the Theory of Bessel Functions, Cambridge University Press, 1962.

[28] X. ZHANG, G. WANG AND Y. WHU, Extensions and sharpenings of Jordan's and Kober's inequalities, J. Ineq. Pure and Appl. Math., 7, (2) (2006), Art. 63. Online: Available online at URL: http: / / jipam.vu.edu.au/article.php?sid=680.

[29] L. ZHU, Sharpening of Jordan's inequalities and its applications, Math. Inequal. Appl., 9, (1) (2006), 103-106.

[30] A. Zygmund, Trigonometric Series, 3rd ed. Cambridge University Press, 2002. 